\title{
6D IONIZATION COOLING CHANNEL WITH RESONANT DISPERSION GENERATION*
}

\author{
Y. Alexahin, K. Yonehara, FNAL, Batavia, IL, R. Palmer, BNL, Upton, NY
}

\section{Abstract}

For muons with preferable for ionization cooling momentum $<300 \mathrm{MeV} / \mathrm{c}$ the longitudinal motion is naturally undamped. In order to provide the longitudinal damping a correlation between muon momentum and transverse position - described in terms of the dispersion function - should be introduced. In the present report we consider the possibility of dispersion generation in a periodic sequence of alternating solenoids (FOFO channel) by choosing the tune in the second passband (i.e. above half-integer per cell) and tilting the solenoids in adjacent cells in the opposite direction. Analytical estimates for equilibrium emittances and cooling rates are presented.

\section{INTRODUCTION}

Obtaining muon beams with high density in 6D phase space is essential for realization of Muon Collider [1], neutrino factories based on accelerated muon beams and other experiments involving muons. Due to short lifetime of muons $(2.2 \mu \mathrm{s})$ the conventional methods of cooling are not applicable, the only method fast enough for cooling muons proposed so far is the ionization cooling (IC).

There are several schemes considered for 6D ionization cooling, the most advanced being so called RFOFO channel [2] (circular or "Guggenheimed") and Helical Cooling Channel [3]. The RFOFO channel is a traditionally looking channel with separate function elements: focusing solenoids, bending magnets, RF cavities, absorbers for actual cooling. It inevitably has large length resulting in decay of about $2 / 3$ of cooled muons.

The Helical Cooling Channel (HCC) is an integrated structure with uniformly distributed along its length solenoidal focusing field, rotating dipole field, gaseous $\mathrm{H}_{2}$ absorber and RF cavities. Ideally it should provide very fast cooling rate owing, in part, to higher RF voltage which can be attained in gas-filled cavities.

However, there is still no practical design for the RF structure which can fit inside a HCC. There is another drawback common to both HCC and "Guggenheimed" RFOFO channel: they are selective to the muon charge sign. This means that $\mu^{+}$and $\mu^{-}$emerging from the pion decay channel should be separated before cooling which will inevitably limit the accepted phase space volume.

Here we present the idea of a 6D cooling channel which can provide almost as fast cooling as in HCC for muons of both signs simultaneously.

\section{IONIZATION COOLING BASICS}

The mechanism of the IC has some similarity with synchrotron radiation damping: particles lose all 3 components of their momentum in the absorber while only the longitudinal component is restored by the RF field. The cooling is distributed between the degrees of freedom as [4]

$$
\begin{aligned}
& \Lambda_{i}=-\frac{d \log \varepsilon_{i N}}{d s}=-g_{i} \frac{d \log E}{\beta^{2} d s}, \\
& \sum_{i} g_{i}=2+g_{L 0} \approx 2\left(\beta^{2}+\frac{1-\beta^{2} / \gamma^{2}}{\ln }\right),
\end{aligned}
$$

where $\ln =\log \left(K \beta^{2} \gamma^{2}\right)>>1, K=2 m_{e} c^{2} / I, I \approx 16 Z^{0.9}[\mathrm{eV}]$ being the ionization potential.

Unlike the SR losses the ionization losses decrease with momentum in the range $p<300 \mathrm{MeV} / \mathrm{c}$ most suitable for cooling. In the result the longitudinal oscillations are naturally anti-damped $\left(g_{L 0}<0\right)$.

In order to overcome this difficulty the muons with higher momentum must make a longer path in the absorber so that they lose more energy. This can be achieved by creating dispersion in particle positions (without significant path lengthening) and using wedge absorbers (RFOFO scheme) or by creating sufficiently large path lengthening with momentum to use a homogeneous absorber (HCC scheme). In the first case the cooling partition numbers are (the transverse modes assumed uncoupled):

$$
\begin{aligned}
& g_{x}=1-w, g_{y}=1, g_{L}=g_{L 0}+w, \\
& w \equiv D_{x} \partial_{x} \log L_{A} .
\end{aligned}
$$

where $L_{A}(x)$ is the wedge absorber width.

Just as in the case of SR the discrete character of ionization interactions leads to a statistical spread in the loss rate (straggling). Besides it there is multiple scattering on the absorber nuclei. These stochastic processes cause heating of the muon beam which counteract cooling. In the result there are some minimum (equilibrium) values of emittances which can be obtained by IC:

$$
\begin{aligned}
& \varepsilon_{\perp N} \approx \frac{m_{e}}{m_{\mu}} \frac{\Sigma_{g}}{\Sigma_{g}-g_{L}} \frac{(Z+\alpha) \beta_{\perp}}{2 \beta}, \\
& \varepsilon_{L N} \approx \frac{m_{e}}{m_{\mu}} \frac{\Sigma_{g}}{g_{L}} \frac{\beta}{8}\left[\beta_{L} \frac{\gamma^{2}+1}{\ln }+\frac{2(Z+\alpha)}{\beta^{2} \beta_{L}} D^{2}\right],
\end{aligned}
$$

where $\beta_{i}$ are beta-functions, parameter $\alpha$ describes electron contribution to multiple scattering, the recent analysis [5] showed that $\alpha<<1$ for muons. The first term in the equation for $\varepsilon_{L N}$ is due to straggling and is usually small compared to the second (scattering) term.

\section{RESONANT DISPERSION GENERATION}

Applying usual formulas for dispersion in the RFOFO ring (or helix)

\footnotetext{
* Work supported by Fermi Research Alliance LLC. Under DE-AC02-
} 07 CH11359 with the U.S. DOE. 


$$
D_{x} \sim \frac{R}{Q_{x}^{2}} \sim \frac{\bar{\beta}_{x}^{2}}{R}, \bar{\beta}_{x}=<1 / \beta_{x}>^{-1},
$$

where $R$ is the ring radius, we see that there is a contradiction between the requirement of small $\beta_{x}$ needed to obtain small $\varepsilon_{x N}$ and the requirement of sufficiently large $D_{x}$ needed for cooling redistribution. In the result the wedge angle has to be very large further reducing the average cooling rate.

It is possible to obtain large dispersion in a periodic structure with alternating transverse field by virtue of the resonant dependence of the periodic orbit on the tune, $x_{\text {p.o. }} \sim 1 / \sin \pi Q_{x}$, and large chromaticity:

$$
D_{x}=\frac{d x_{\text {p.o. }}}{d \delta_{p}} \approx-\pi Q_{x}^{\prime} x_{\text {p.o. }} \cot \left(\pi Q_{x}\right)
$$

Noticing that naturally the chromaticity is negative, $Q_{x}{ }^{\prime}<0$, we can obtain also large positive momentum compaction factor if the tune is just above an integer.

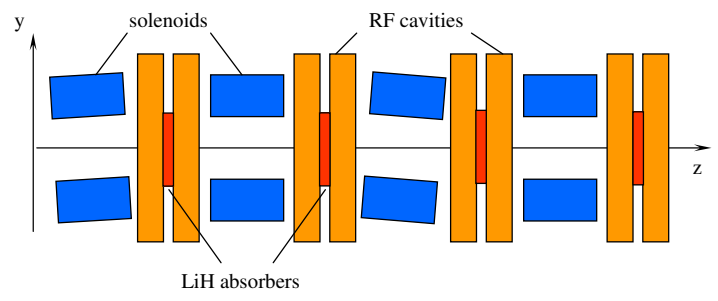

Figure 1. Schematic of the FOFO channel cell

\section{FOFO CHANNEL WITH TILTED SOLENOIDS}

The basic cell of the simplest channel considered for ionization cooling - the FOFO channel - consists of two solenoids with opposite polarity. In principle, the conditions for resonant dispersion generation can be realized by choosing the phase advance per cell $\mu \geq 2 \pi$ and tilting the solenoids in the same direction. However, in this case the phase advance over one solenoid is $\sim \pi$ so that too large a tilt angle is necessary.

The dispersion can be created more efficiently by choosing $\mu \geq \pi$ and tilting the solenoids of the same polarity in adjacent cells in opposite directions. In the result two cells form a super-cell (Fig.1) with total phase advance $2 \mu>2 \pi$ so that the resonance condition is fulfilled again.

With every other solenoid tilted in the same plane (e.g. vertical) the channel has the reflection symmetry w.r.t. this plane. Therefore both $\mu^{+}$and $\mu^{-}$can propagate in it (shifted by $\pi$ in the RF phase) along periodic orbits which are mirror reflections of each other.

Another important feature of the channel is a moderate beta-beat which permits to fill the channel (including RF cavities) with gaseous absorber as it is assumed for the HCC. However, the momentum compaction factor may be not large enough to ensure the longitudinal cooling so that some wedge absorber will be necessary in addition to $\mathrm{GH}_{2}$.

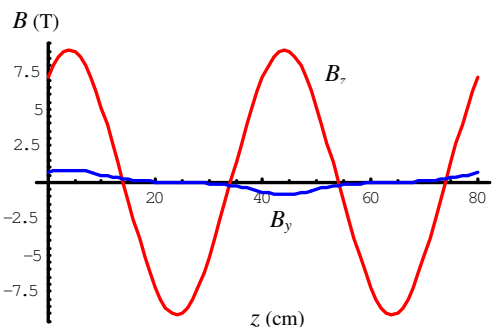

Figure 2. Magnetic field along the channel

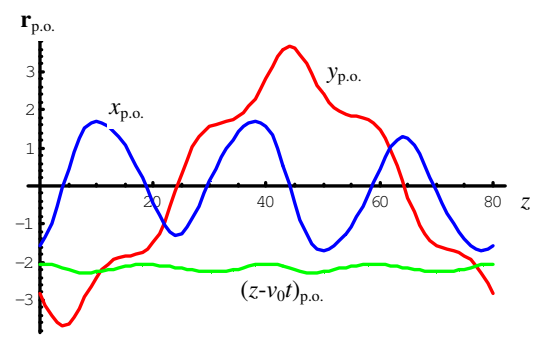

Figure 3. Periodic orbit in the channel

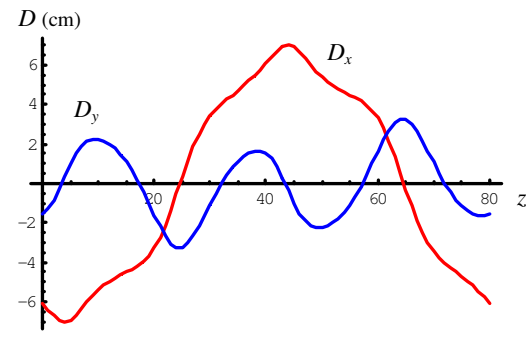

Figure 4. Dispersion functions along the channel

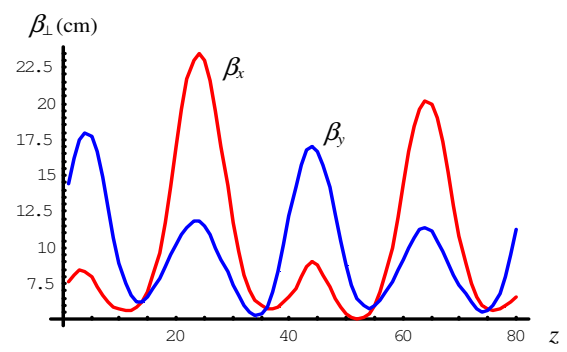

Figure 5. $\beta$-functions along the channel

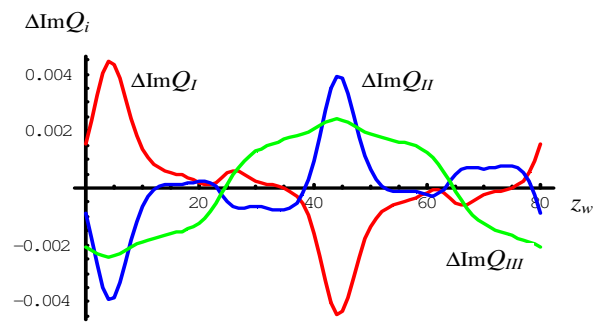

Figure 6. Change in cooling decrements vs wedge absorber position

Here we consider the last section of 6D IC channel employing $800 \mathrm{MHz}$ RF. The basic parameters of the channel: double cell of length $80 \mathrm{~cm}$ consists of four $8 \mathrm{~cm}$ long solenoids with inner and outer radii $10 \mathrm{~cm}$ and $16 \mathrm{~cm}$ respectively. The first and third solenoids are tilted vertically by angle $\pm 65 \mathrm{mrad}$. With current density $\sim 450 \mathrm{~A} / \mathrm{mm}$ (well within the reach of high-temperature 
superconductors) the maximum on-axis longitudinal field is 9.5T. The distribution of magnetic field along the channel axis (with contribution from 8 more solenoids on each side of the considered cell taken into account) is shown in Fig. 2.

Two-cell pillbox RF cavities of length $2 \times 4 \mathrm{~cm}$ are placed between each pair of solenoids. It is assumed that filling with $\mathrm{GH}_{2}$ of $19 \%$ density of $\mathrm{LH}_{2}$ will allow to raise the amplitude field up to $50 \mathrm{MV} / \mathrm{m}$. $\mathrm{LiH}$ wedge absorbers are placed between the cells of RF cavities (see discussion below).

\section{Algorithm for the IC channel design}

To compute and optimize parameters of IC channels a new program is being created - Methodical Ionization Cooling Channel Design (MICCD) - which was used in this particular case. It performs the following calculations: - periodic orbit and eigenvectors of the transfer matrix with account of (regular part of) ionization losses and feeddown effect from nonlinear fields;

- emittance growth due to scattering and straggling, equilibrium values (if exist);

- regular nonlinear dynamics: dependence of tunes and damping rates on the amplitudes, resonance excitation;

- nonlinear corrections to equilibrium emittances.

\section{Linear optics and emittances}

Figs. 3 and 4 show the $\mu^{+}$periodic orbit and dispersion while Fig. 5 presents the effective beta-functions which are the sums of the Mais-Ripken functions [6] describing projection of the normal modes onto the horizontal and vertical planes: $\beta_{x, y}=\beta_{x, y I}+\beta_{x, y I I}$.

For the generic design the initial tunes (determined by on-axis fields) were chosen sufficiently far from the integer $\left(Q_{\mathrm{I}, \mathrm{II}} \approx 1.25\right)$ so that the wedge absorbers are necessary. Without them the complex tunes of the normal modes of oscillations w.r.t. the periodic orbit are: $Q_{\mathrm{I}, \mathrm{II}, \mathrm{III}}=1.4242+0.0084 \mathrm{i}, \quad 1.505+0.0063 \mathrm{i}, \quad 0.2105-0.0052 \mathrm{i}$. The oscillation amplitudes are reduced by $\exp \left(2 \pi \operatorname{Im} Q_{i}\right)$ per one cell that means anti-damping for the $3^{\text {rd }}$ mode.

According to Fig. 4 the dispersion reaches maximum at the center of tilted solenoids. However, these are not the best places for wedge absorbers: the beta-functions have maximum there as well which means their large contribution to equilibrium emittances. To find the best location for wedge absorbers a scan has been performed. Fig. 6 shows the contribution from a horizontal $\mathrm{LiH}$ wedge with angle $0.1 \mathrm{rad}$ to the cooling decrements of the normal modes (vertical wedge is much less efficient). One can see that if the wedge is placed at locations of maximum dispersion it redistributes cooling partition between the transverse modes more than it gives to the longitudinal mode.

The best locations for wedges are between the cells of RF cavities at 14, 34, 54 and $74 \mathrm{~cm}$. To equalize the cooling decrements they should have different angle: 0.32 rad and $0.2 \mathrm{rad}$ alternatingly. To reduce the wedge width at the beam position their apexes are shifted by $\pm 0.5 \mathrm{~cm}$ from the axis. The resulting tunes, decrements and equilibrium emittances are cited in Table 1.

Table 1. Normal mode complex tunes and emittances.

\begin{tabular}{|l|l|l|l|}
\hline & mode I & mode II & mode III \\
\hline$Q$ & $1.4184+.0048 \mathrm{i}$ & $1.5067+.0052 \mathrm{i}$ & $0.1863+.004 \mathrm{i}$ \\
\hline$\varepsilon_{N}(\mathrm{~mm})$ & 0.29 & 0.30 & 0.29 \\
\hline
\end{tabular}

\section{Nonlinear effects}

The inevitable consequence of strong focusing in cooling channels is large angular divergence which enhances the effect of nonlinearity in relativistic kinematics. Another source of aberrations are nonlinear components of magnetic fields which in the considered channel can be to some extent adjusted by playing with solenoid tilts and offsets so as to cancel the kinematical effect.

The analysis of nonlinear effects has been performed on the basis of Kamel's extension of Deprit's algorithm for non-Hamiltonian systems [7]. Table 2 presents derivatives of the normal mode tunes w.r.t. the action variables $J_{i}$. One can see that cooling of all modes fails when their respective $J_{i}$ are about $1 \mathrm{~cm}$. The corresponding Courant-Snyder invariants are $2 \mathrm{~cm}$ so the cooling aperture is about $8 \sigma$.

Table 2. Complex detuning coefficients

\begin{tabular}{|l|c|c|c|}
\hline & $\partial Q_{\mathrm{I}}$ & $\partial Q_{\mathrm{II}}$ & $\partial Q_{\mathrm{III}}$ \\
\hline$/ \partial J_{\mathrm{I}}$ & $-.0039-.0047 \mathrm{i}$ & $.0964+.0059 \mathrm{i}$ & $.1226-.0015 \mathrm{i}$ \\
\hline$/ \partial J_{\mathrm{II}}$ & $.0838+.0120 \mathrm{i}$ & $.2775-.0052 \mathrm{i}$ & $.1112-.0057 \mathrm{i}$ \\
\hline$/ \partial J_{\mathrm{III}}$ & $.0613+.0054 \mathrm{i}$ & $.0552+.0030 \mathrm{i}$ & $-.1081-.0039 \mathrm{i}$ \\
\hline
\end{tabular}

The presented analysis is a preliminary one, it should be verified (and perfected) by tracking simulations with codes like ICOOL and G4BL.

\section{REFERENCES}

[1] C. Ankenbrandt et al. PRSTAB 2, 081001 (1999).

[2] R. Palmer et al. PRSTAB 8, 061003 (2005).

[3] Y. Derbenev, R. Johnson PRSTAB 8, 041002 (2005).

[4] D. Neuffer, NIM A 532, 26-31 (2004).

[5] A. Tollestrup, LEMC Workshop FNAL (2006) http://www.muonsinc.com/

[6] G. Ripken, F. Willeke, "Methods of Beam Optics", DESY 88-114 (1988).

[7] L. Michelotti, Intermediate Classical Dynamics with Applications to Beam Physics, (John Wiley \& Sons, Inc., New York, 1995). 